\title{
On the Many Applications of Nanometer-Thin Pure Boron Layers in IC and Microelectromechanical Systems Technology
}

\author{
Lis K. Nanver ${ }^{1,3, *}$, Tihomir Knezevic², Xingyu Liu¹, Shivakumar D. Thammaiah ${ }^{3}$, and Max Krakers ${ }^{1}$ \\ ${ }^{1}$ MESA + Institute for Nanotechnology, University of Twente, 7522NH Enschede, The Netherlands \\ 2 University of Zagreb, Faculty of Electrical Engineering and Computing, MINEL, 10000 Zagreb, Croatia \\ ${ }^{3}$ Department of Materials and Production, Aalborg University, 9220 Aalborg, Denmark
}

\begin{abstract}
An overview is given of the many applications that nm-thin pure boron (PureB) layers can have when deposited on semiconductors such as $\mathrm{Si}, \mathrm{Ge}$, and $\mathrm{GaN}$. The application that has been researched in most detail is the fabrication of $n$ m-shallow $p^{+} n$-like Si diode junctions that are both electrically and chemically very robust. They are presently used commercially in photodiode detectors for extremeultraviolet (EUV) lithography and scanning-electron-microscopy (SEM) systems. By using chemicalvapor deposition (CVD) or molecular beam epitaxy (MBE) to deposit the $\mathrm{B}$, PureB diodes have been fabricated at temperatures from an optimal $700{ }^{\circ} \mathrm{C}$ to as low as $50{ }^{\circ} \mathrm{C}$, making them both front- and back-end-of-line CMOS compatible. On Ge, near-ideal $p^{+} n$-like diodes were fabricated by covering a wetting layer of $\mathrm{Ga}$ with a PureB capping layer (PureGaB). For GaN high electron mobility transistors (HEMTs), an Al-on-PureB gate stack was developed that promises to be a robust alternative to the conventional Ni-Au gates. In MEMS processing, PureB is a resilient nm-thin masking layer for Si micromachining with tetramethyl ammonium hydroxide (TMAH) or potassium hydroxide $(\mathrm{KOH})$, and low-stress PureB membranes have also been demonstrated.
\end{abstract}

Keywords: Pure Boron, Chemical Vapor Deposition (CVD), Molecular Beam Epitaxy (MBE), Photodiodes, Silicon Micromachining.

\section{CONTENTS}

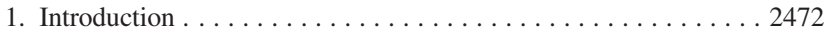

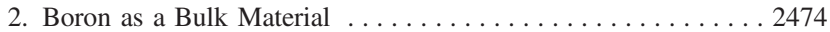

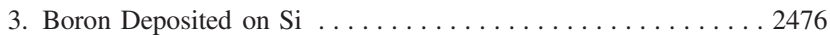

4. Boron Deposited on Non-Si Semiconductors . . . . . . . . . . . . 2478

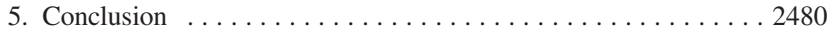

Acknowledgments . . . . . . . . . . . . . . . . . . . 2480

References and Notes .................... 2480

\section{INTRODUCTION}

The drive towards smaller and smaller devices for integrated circuits has triggered a race to develop materials that will allow high performance in devices of only a few nanometer in size. Graphene has led the race, promising high mobility and high material strength in a $2 \mathrm{D}$ sheet of well-organized carbon atoms [1]. However, disappointments with respect to manufacturability and bandgap management have shifted research efforts to a series of other,

\footnotetext{
*Author to whom correspondence should be addressed.
}

electronically promising, 2D materials, some of which have been successfully fabricated such as silicene [2] germanene [3], molybdenum disulfide $\left(\mathrm{MoS}_{2}\right)$ [4], phosphorene [5], and borophene [6]. While the properties of these 2D materials are exciting, they all face major challenges as far as manufacturability is concerned, not the least because they are difficult to fabricate, and their properties change when they are patterned or brought into contact with other materials. Therefore, attention is also being directed towards interfaces where 2D sheets of compounds can be created with special bonding structures that are not attainable in the form of a bulk material.

As an example of an interface that has proven to have unexpectedly attractive properties, this paper will give an overview of pure boron (PureB) depositions on silicon that create nm-shallow $p^{+}$-type regions even when no doping of the bulk Si is expected. The exact origin of the special PureB behavior is still under investigation but an overwhelming experimental evidence suggests that the B-to$\mathrm{Si}$ bonds create a layer of negative fixed charge which 


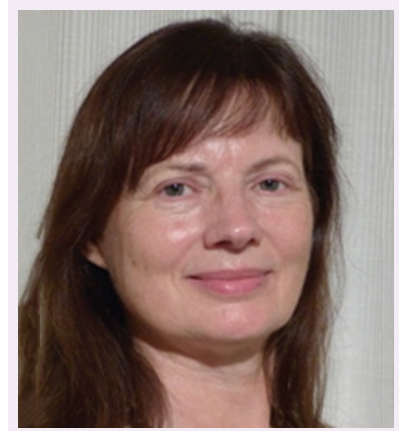

Lis K. Nanver received a master's degree in physics from the University of Aarhus, Denmark, in 1979, and a docteur ingenieur degree in physics applied to telecommunications from the Ecole Nationale Supérieure des Télécommunications (ENST), Paris, France, in 1982, and a Ph.D. degree in electrical engineering from Delft University of Technology, The Netherlands, in 1987. From 1987-2018 she was a researcher at Delft University of Technology, from 2001 as professor. Since 2015 she has also been guest professor at University of Twente, Enschede, The Netherlands, and Aalborg University, Aalborg, Denmark. Her main research interests are new devices and integration processes, mainly for RF, microwave, or smart sensor applications. She has pioneered several new technologies such as substrate transfer for true two-sided contacting, and ultrashallow junction diodes using laser annealing/solid-phase epitaxy. Her research on new pure-dopant CVD processes for creating extremely shallow diodes (PureB for Si and PureGaB for Ge devices) has resulted in several leading-edge applications such as high-linearity silicon-on-glass varactor diodes, Si photodiode detectors for low penetration-depth beams and low-leakage Ge-on-Si photodiodes. Professor Nanver is associate editor for IEEE Electron Device Letters and among others she has served on the committees of BCTM and ESSDERC.

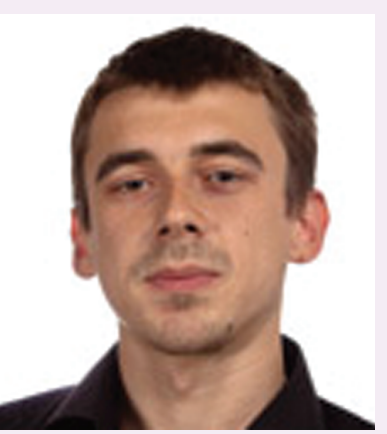

Tihomir Knezevic received his master and summa cum laude Ph.D. in electrical engineering from the University of Zagreb, Croatia in 2009 and 2017, respectively. Currently, he is a postdoctoral researcher at the University of Zagreb, Croatia, and a guest researcher at the University of Twente, The Netherlands. He has participated on more than 15 research projects funded by national and international research agencies and international industrial partners, contributing with his expertise in characterization, modeling and simulation of advanced semiconductor structures. His focus has been on photodiodes for (vacuum-) ultraviolet light and low-energy electron detectors, drift-detector structures, and (singlephoton) avalanche detectors. In particular, he performed electrical, optical and physical characterization of PureB layers and devices which led to the construction of a functional model for these devices. This work has contributed to more than 25 papers in journals and conference proceedings, 4 of which received best paper awards including the 2010 IEDM Roger Haken Best Student Paper Award. He will continue this line of work, among others in the 2020-2021 Fulbright Visiting Research U.S. scholar program.

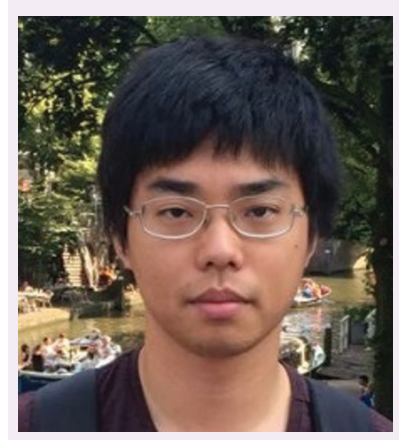

Xingyu Liu was born in Liaoning, China, in 1988. He received the B.S. degree in electrical and information engineering from Dalian University of Technology, Dalian, China, in 2012, and the M.S. degree in microelectronics from the Delft University of Technology, Delft, the Netherlands, in 2014. He is currently pursuing the Ph.D. degree in the Integrated Devices and Systems group, University of Twente, the Netherlands. His research interests include semiconductor device processing and optoelectronic characterization, particularly for silicon-based detectors, imagers and light-emitting diodes.

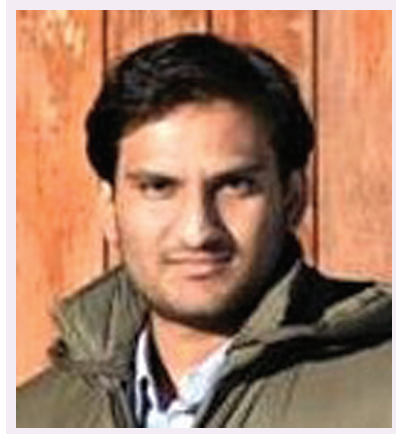

Shivakumar D. Thammaiah received M.S degree in Materials Science from Jawaharlal Nehru Centre for Advanced Scientific Research (JNCASR), Bengaluru, India in 2015. He is currently pursuing his Ph.D. degree from Aalborg University, Aalborg, Denmark and is also a visiting research student at University of Twente, Enschede, The Netherlands. His research interests include development, fabrication and characterization of contacts and diffusion barriers in $\mathrm{Si}$ and $\mathrm{GaN}$ based devices. 


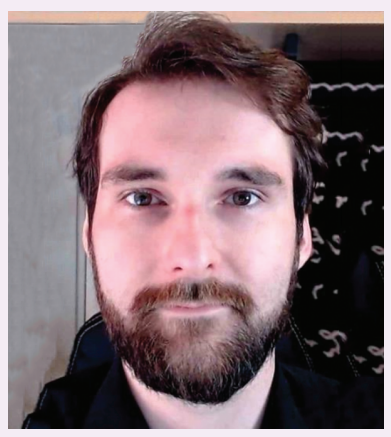

Max Krakers received his B.Sc. in Advanced Technology in 2016 and attained his M.Sc. in Nanotechnology in 2018 both from the University of Twente. He joined the integrated Devices and Systems group in 2018 as a research assistant working on PureB diodes, which became the topic of his Ph.D. research in 2020.

attracts holes to the interface [7]. The charge concentration is so high that a steep electrical field gradient up to the interface efficiently suppresses the injection of electrons from the $n$-substrate [8]. This results in junction formation with low saturation currents resembling that of ideal deep $p^{+} n$ junctions. Together with an exceptional robustness of both the PureB interface to the $\mathrm{Si}$ and the bulk boron layer itself, these properties have been pivotal for already commercialized application to photodiodes for high-dose beams with low-penetration depths in $\mathrm{Si}$, such as vacuumultraviolet light and low-energy electrons [9, 10].

To our knowledge, the PureB $p^{+} n$-like diodes are the first example of bulk boron having an electrical function in silicon devices. Historically, boron has been, and still is, the main $p$-type dopant in Si IC-technology. To follow Moore's Law and continually reduce junction depths, the industry has transitioned from initially high-temperature diffusion techniques from e.g., boron-nitride wafers, to implantation/annealing of $\mathrm{B}^{+}$or $\mathrm{BF}_{2}^{+}$, and finally to chemical-vapor deposition (CVD) deposition of B-doped Si layers. Doping from CVD deposited pure B layers was also considered but any deposits of pure boron layers were regarded as undesirable parasitics $[11,12]$. In c-Si solarcell fabrication, doping from borosilicate-glass layers is still commonly used. In this application, boron-rich layers formed at the $\mathrm{Si}$ surface reduce carrier lifetime and are necessarily removed [13]. The name "PureB" diodes was introduced to underline that the attractive characteristics of these diodes are the result of a surface coverage of pure B and not B doping of the bulk Si. The PureB layers discussed in this paper were fabricated from $250{ }^{\circ} \mathrm{C}$ to $700{ }^{\circ} \mathrm{C}$ by $\mathrm{CVD}$ but electrically active interfaces grown in a molecular beam epitaxy (MBE) system were also fabricated at temperatures down to $50{ }^{\circ} \mathrm{C}$ [14].

While the bulk of the research has been directed towards PureB Si devices, interesting applications of pure B depositions in $\mathrm{Ge}$ and $\mathrm{GaN}$ diodes have also been identified. In these applications it is the high strength of the B-B bond [15] that has been the differentiating factor. In both cases the PureB layer forms a barrier to chemical interaction of Al-metallization with the semiconductor, thus offering a means of using $\mathrm{Al}$ to replace less CMOS-compatible metals commonly used to fabricate contacts and diodes in these non-Si semiconductors. On Ge, the role of providing an interfacial hole layer with $p^{+}$-like properties was taken over by a wetting-layer of gallium, another group III atom, which was capped with a layer of B as barrier to Al metallization. With this "PureGaB" process, low dark-current photodiodes were fabricated and operation as single-photon avalanche diodes (SPADs) was demonstrated [16]. For GaN HEMTs, an Al-on-PureB gate stack was developed that promises to be a robust alternative to the conventional $\mathrm{Ni}-\mathrm{Au}$ gates [17]. In microelectromechanical systems (MEMS) processing, PureB functions as a resilient $\mathrm{nm}$-thin masking layer for $\mathrm{Si}$ micromachining with tetramethyl ammonium hydroxide (TMAH) or potassium hydroxide $(\mathrm{KOH})$, and low-stress PureB membranes have also been demonstrated [18]. This paper gives an overview of these diverse applications of PureB depositions with focus being placed on the material properties.

\section{BORON AS A BULK MATERIAL}

As a bulk material, boron has some very special properties. As the lightest of the semimetals, next to carbon in the periodic table, boron is the element with the lowest atomic number to form extended covalent networks. Unlike carbon with its 4 valence electrons in the outer shell, B has only 3 and cannot form a closed-shell electronic structure through simple 2-electron covalent bonds [19]. Instead, boron forms highly delocalized bonds, sharing electron pairs among three (or more) atoms. As a result, 3D bulk B structures are quite complex but even in an amorphous form they may contain regular B12 icosahedra, irregularly bonded to each other [20].

Most of the $\mathrm{B}$ layers discussed in this paper were grown from diborane $\left(\mathrm{B}_{2} \mathrm{H}_{6}\right)$ by $\mathrm{CVD}$ in commercial systems as described in detail in Refs. [21, 22]. Layers of a single $\mathrm{nm}$ to tens of $\mathrm{nm}$ in thickness were deposited in the temperature range from $250{ }^{\circ} \mathrm{C}$ to $700{ }^{\circ} \mathrm{C}$. For temperatures below $400{ }^{\circ} \mathrm{C}$ the layers are thought to be amorphous with a high hydrogen content but in all cases the structural composition is unknown. While most research effort has gone into studying the electrical properties of diodes made with these depositions, the bulk B layers also had attractive mechanical/chemical properties that are related to the high strength of the B-B bonds. This was first realized for the application as PureB-only radiation-entrance windows 

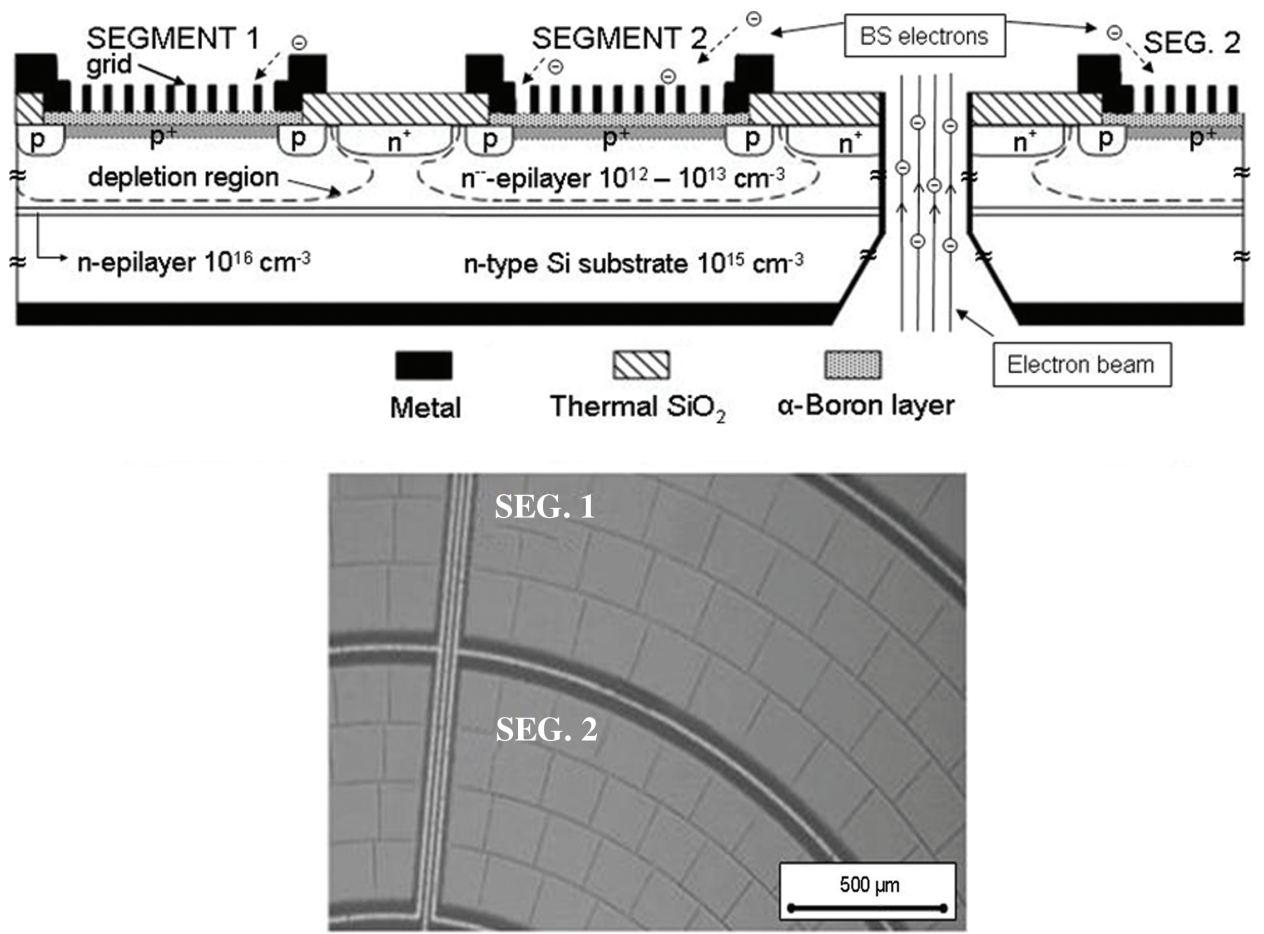

Figure 1. Schematic cross-section (top) and micro-image (bottom) of two neighboring segments of a PureB Si photodiode detector for SEM systems. A through-wafer hole has been etched by micromachining in the $\mathrm{Si}$ as aperture for the electron beam. The depletion of the typically $40 \mu \mathrm{m}$ deep $n^{--}$ epitaxial layer is indicated. Diode segments are isolated by the $n^{+}$channel stop and the undepleted $n^{--}$layer. An Al-metal grid has been patterned directly on the PureB. Reprinted with permission from [10], Šakić, A., et al., 2010. Versatile Silicon Photodiode Detector Technology for Scanning Electron Microscopy with High-Efficiency sub-5 keV Electron Detection. Proc. 2010 IEEE Intl. Electron Devices Meeting, Washington, DC, USA, pp.31.4.1-31.4.4. Copyright@IEEE.

for low-energy electron Si photodiodes. Layers as thin as $2 \mathrm{~nm}$ were found to be chemically very inert, allowing metallization with pure Al that could be locally removed in diluted HF which does not etch the B-layer [10]. This enabled the integration of complex PureB Si detector circuits that displayed exceptional robustness and stability under exposure to high-dose irradiation and harsh environments such as those found in extreme-ultraviolet (EUV) lithography equipment [23]. For electron detectors, besides the very limited thickness of the PureB layers, the transmissivity is also enhanced by the fact that $\mathrm{B}$ has a low atomic number which minimizes the scattering of the electrons [24]. Electrons with energies down to $200 \mathrm{eV}$ were reliably detected [25], and PureB detectors have become popular for use in scanning- and transmission-electronmicroscopy (SEM/TEM) systems [10]. An example is given in Figure 1.

PureB was also found to be resistant to both TMAH or $\mathrm{KOH}$, anisotropic Si-etchants that are widely used for fabricating MEMS [26]. As masks for the etching of Si, the B-layers displayed excellent adhesion, conformality and etch selectivity $\left(>10^{4}\right)$ at low stress levels that could be varied by changing the deposition conditions. As opposed to the more commonly used dielectric masking layers, e.g., silicon dioxide and silicon nitride $\left(\mathrm{SiN}_{\mathrm{x}}\right)$, PureB as thin as $2 \mathrm{~nm}$ was sufficient for complete protection of the Si. Patterning of the boron was achieved by standard resist masking and removal by aluminum etchant. Reducing the deposition temperature to $300{ }^{\circ} \mathrm{C}$ degraded the quality of the PureB layer in terms of surface coverage and compactness, resulting in a reduction of selectivity and a higher risk of weak spots. Therefore, masking of standard TMAH Si-etching was no longer possible with a 2-nm-thick layer [18, 27]. However, just like conventional PECVD masking layers, etch-susceptible weak spots in the PureB layer could be eliminated by simply depositing a thicker layer. With a thickness of $15 \mathrm{~nm}$, excellent etch results were achieved as shown in Figure 2. In addition, these $300{ }^{\circ} \mathrm{C}$ PureB layers displayed near-zero stress [27] which, along with a thickness in the nm range and the low deposition temperature, make PureB a very attractive layer for micromachining applications and as a back-end CMOS compatible process.

Both suspended and closed PureB membranes were demonstrated, which is of interest for a number of applications. In SEM systems, commercial protection membranes are commonly made of $\mathrm{SiN}, \mathrm{SiC}, \mathrm{SiO}_{2}$ and polyimide, but materials such as graphene are being studied due to the low atomic number, the monolayer thickness, the high mechanical stiffness, and high electrical conductivity which would eliminate parasitic charging effects and artefacts common for standard dielectric membranes [28, 29]. 

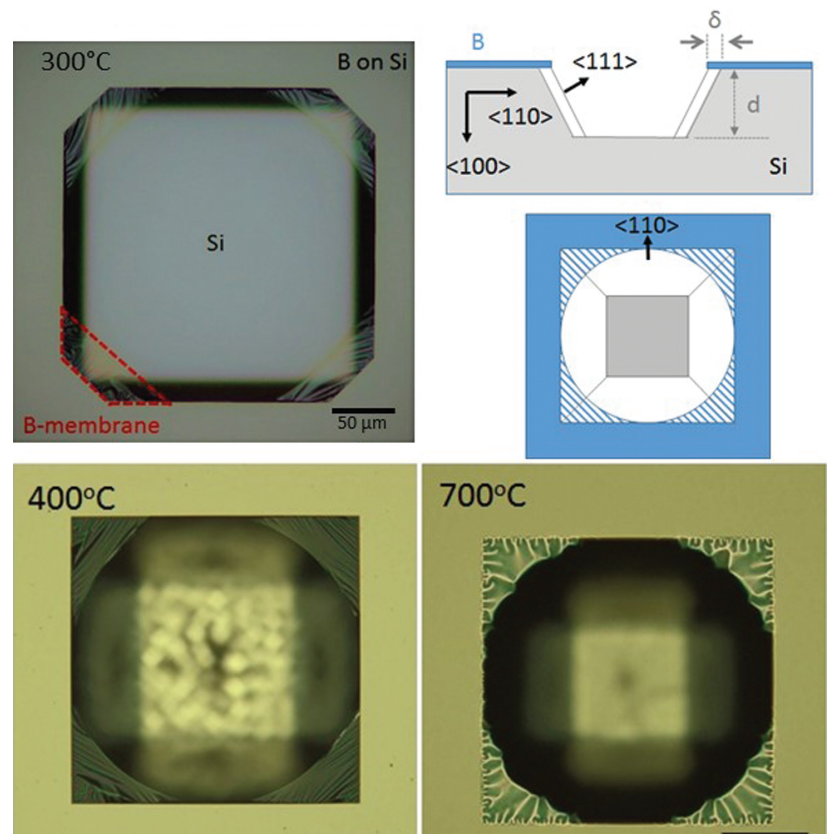

Figure 2. Schematic showing the undercut, $\delta$, in $<110\rangle$ direction and cavity depth, d, when $\mathrm{Si}$ is etched with TMAH through a window in a PureB masking layer. Optical microscope images of PureB membranes left standing in the corners of the inverted pyramids etched in $\mathrm{Si}$ with TMAH though a circular window patterned in a PureB mask; for B deposition at $300{ }^{\circ} \mathrm{C}, 400{ }^{\circ} \mathrm{C}$ and $700{ }^{\circ} \mathrm{C}$ to a thickness of $15 \mathrm{~nm}, 6 \mathrm{~nm}$, and $13 \mathrm{~nm}$, respectively. A schematic top view is shown of the membranes and the original circular window in the PureB mask. Reprinted with permission from [18], Liu, X., et al., 2017. Nanometer-thin pure boron layers as mask for silicon micromachining. J. Micromechanical Systems, 26(6), pp.1428-1434. Copyright@IEEE. and from [27], Liu, X., et al., 2019. Micromachined nanometer-thin boron membranes on silicon. Materials Research Express, 6(11), p.116438. Copyright@IOP Publishing.

PureB membranes also have many of these advantages: $\mathrm{B}$ has an even lower atomic number than $\mathrm{C}$ and the CVD layers are conductive. Although the resistivity is very high [30], it is still sufficient for preventing charge accumulation [31]. An important advantage with respect to graphene is that PureB thin films are highly manufacturable $[9,10]$. In particular, all PureB layers have high front-end CMOS compatibility and those deposited below $450{ }^{\circ} \mathrm{C}$, can be integrated in complex process flows that include fully-processed CMOS circuits.

The proven resistance of PureB to EUV exposure and cleaning plasmas [9] has meant that boron has also been studied as a material for capping extreme-ultraviolet (EUV) pellicles for EUV lithography systems [32]. These pellicles are preferably made of polysilicon due to the high transmissivity and mechanical strength. For highvolume EUV exposure, the infrared emission needed for preventing local overheating of the pellicle requires capping of the polysilicon with layers of suitable emissivity. Capping layers incorporating boron were found to have both good transmissivity [33] and emissivity [32]. To extend this to using pure B membranes for pellicles, high demands on the mechanical strength and stiffness need to be met. Tensile stress is needed to prevent sagging, but film shrinkage due to densification during exposure/heating gives a risk of excess tension and consequent deformation/breakage. Films with maximum material density are therefore preferred. The PureB grown at $700{ }^{\circ} \mathrm{C}$ was found to be the most compact material which no doubt is a result of the high mobility of the deposited B atoms along the $\mathrm{Si} / \mathrm{B}$ surface before adhesion.

In the last 4 years, there have been several reports of the synthesis of borophene by CVD [34, 35] among others on silver or copper. Extended one-atom-thick boron sheets were constructed from planar hexagonal 36-atom clusters of B [36]. As theoretically predicted, these were buckled 2D sheets with metallic characteristics. They are also predicted to have high electrical and heat conductivity, and to be more flexible and stronger than graphene. Moreover, these properties are theoretically predicted to be "tunable," depending on the material's orientation and different arrangements of vacancies in the crystal structure [37]. So, it is not without reason that research interest in borophene is on the rise. However, a fabrication method allowing large quantities to be made still needs to be found. Moreover, borophene has high reactivity which may be attractive for battery application [37] but a disadvantage for application as protection membranes. A rapid oxidation of the boron in open air has been reported [37]. In contrast, for the PureB capping of detectors for SEM systems there has been no indication that the B surface is prone to oxidation, something which would negatively impact the signal gain of the photodiodes. Nevertheless, experiments have shown that contamination of the CVD system will readily lead to incorporation of oxygen in the B-layer [38], a danger that increases as the deposition temperature, and thus the deposition rate, is reduced. For synthesis of borophene, such contamination has been reported to be devastating. Unlike PureB, this and the many other manufacturability challenges mean that borophene is very far from entering any products.

\section{BORON DEPOSITED ON SI}

The electrical behavior of PureB Si diodes is quite clearly related to the conditions at the interface with $\mathrm{Si}$, but just as for bulk B, the exact bonding structure is unknown. Nevertheless, the fact that $B$ readily is activated as a p-dopant impurity in bulk Si suggests that a similar mechanism for binding electrons from the $\mathrm{Si}$ to the $\mathrm{B}$ could be effective at the interface. The fact that depositions of $\mathrm{Ga}$, also a group III p-dopant in $\mathrm{Si}$, have been shown to also create $p^{+} n$-like junctions on $\mathrm{Si}$ [39], also suggests that the dopant nature of $\mathrm{B}$ and $\mathrm{Ga}$ is the important feature. For the diode performance the differentiating factor is that the concentration of holes resulting from such "interface doping" appears to be much higher than anything achievable by conventional thermal doping of the Si. At $700{ }^{\circ} \mathrm{C}$, the solid solubility of $\mathrm{B}$ in $\mathrm{Si}$ is $2 \times 10^{19} \mathrm{~cm}^{-3}$ but from experiments with 
$500{ }^{\circ} \mathrm{C}-700{ }^{\circ} \mathrm{C}$ PureB p-regions used as emitters in pnp transistors (Fig. 3) the emitter efficiency corresponded to a total doping of about $10^{15} \mathrm{~cm}^{-2}$. This is equivalent to an extremely high surface doping concentration of around $10^{22} \mathrm{~cm}^{-3}$ [21]. The emitter efficiency is a measure for how well the p-region suppresses the injection of minority carrier electrons from the substrate, and much of the experimental work has been dedicated to determining the resulting electron current [40]. The deposition conditions have a large influence with the temperature being the most important parameter. As the temperature is lowered, the electron current increases to be about 10 times higher at $400{ }^{\circ} \mathrm{C}$ than at $700{ }^{\circ} \mathrm{C}$. On the other hand, the deposition rate becomes more and more important as the temperature is lowered because the mobility of the B atoms along the $\mathrm{Si}$ surface decreases dramatically. This makes it less likely that a complete surface coverage of B will be formed, and if the deposition rate is too fast, overgrowth of the initially deposited $\mathrm{B}$ becomes imminent. At the same time, the bulk B will also be less compact, and this can become
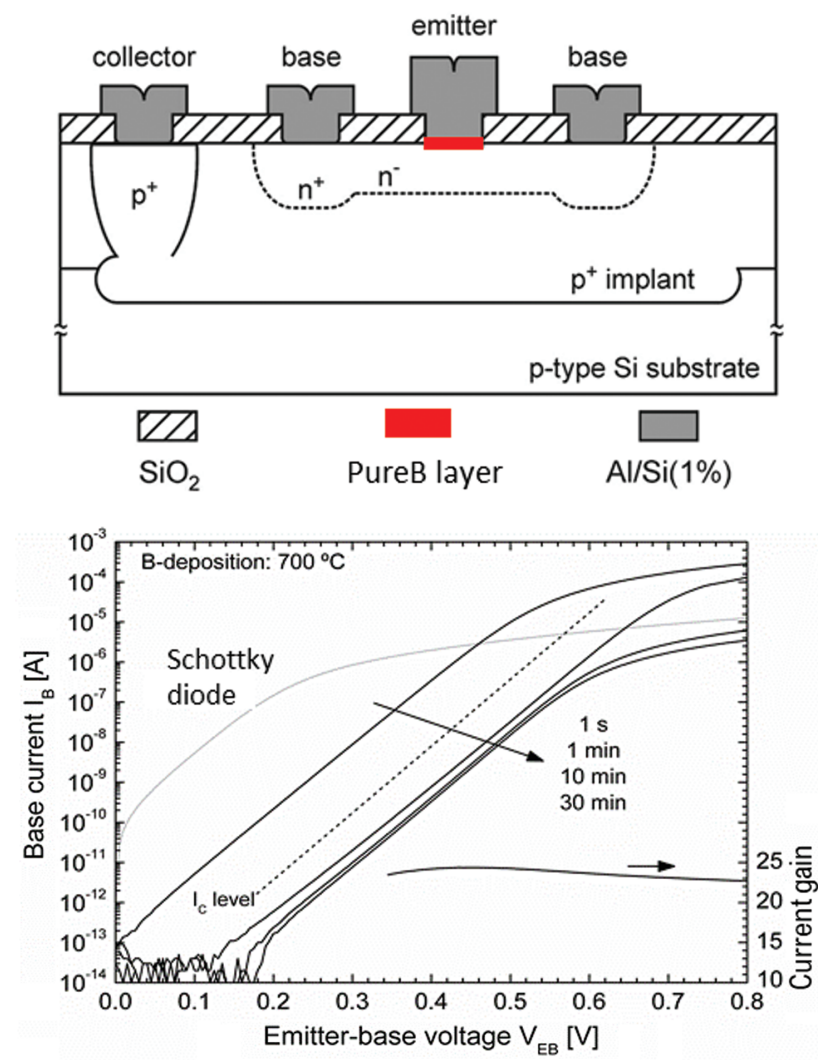

Figure 3. Top: Schematic cross sections of vertical pnp bipolar transistors fabricated with PureB emitters. Bottom: Base current of pnp transistors for different B-deposition times at $700{ }^{\circ} \mathrm{C}$. The collector current level is also shown, as is the common-emitter current gain for the 30 -min deposition time. The emitter area is $40 \times 40 \mu \mathrm{m}^{2}$ [21]. Reprinted with permission from [21], Sarubbi, F., et al., 2010. High effective gummel number of CVD boron layers in ultrashallow $p^{+} n$ diode configurations. IEEE Transactions on Electron Devices, 57(6), pp.1269-1278. Copyright@IEEE. problematic when metal layers need to be applied. Weak spots in the B structure may allow the metal to get too close to or even touch the $\mathrm{Si}$, both of which will increase the electron current [7]. A structural and electrical comparison of the $400{ }^{\circ} \mathrm{C}$ and $700{ }^{\circ} \mathrm{C}$ PureB layers is shown in Figure 4.
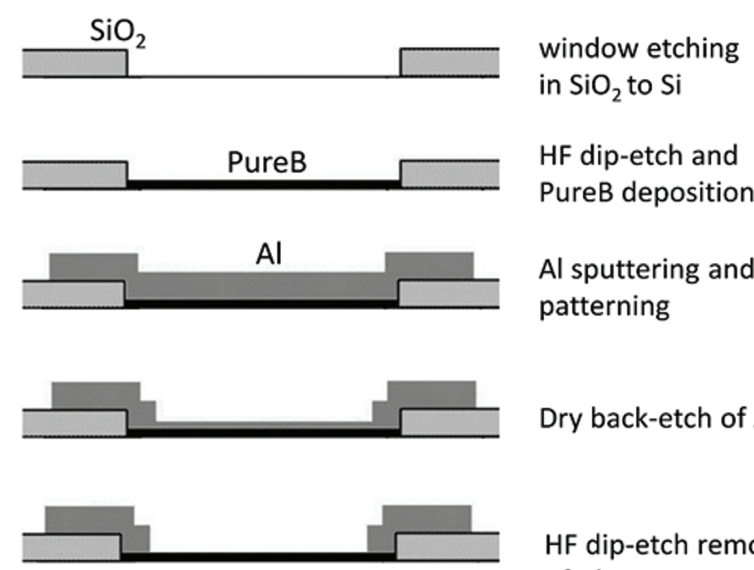

Dry back-etch of Al

$\mathrm{n}-\mathrm{Si}$

HF dip-etch removal of $A$ l to PureB
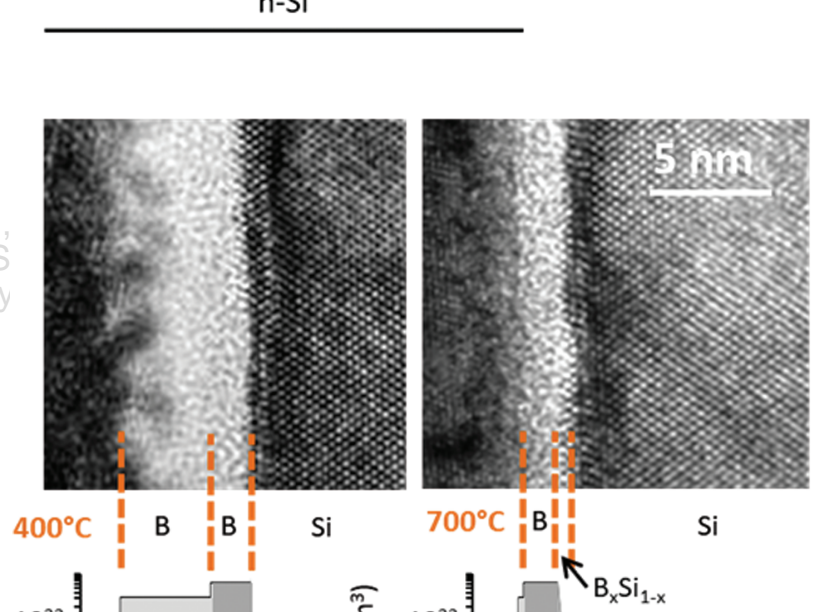

Si

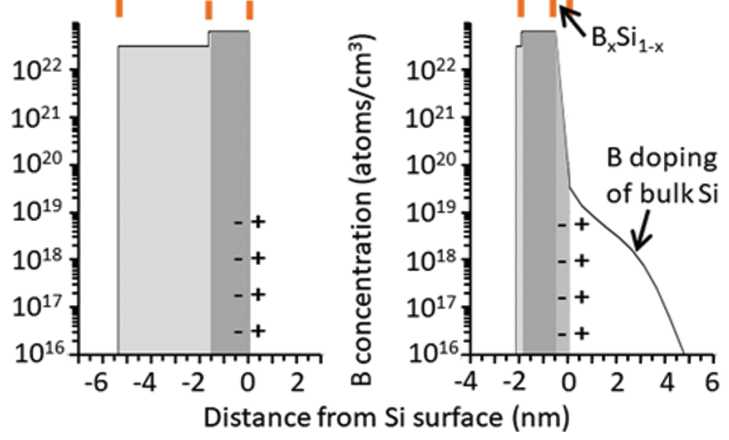

Figure 4. Top: Schematic of the basic process flow for fabricating photodiodes with PureB-only light-entrance windows. Middle: HRTEM images of PureB layers grown at $400{ }^{\circ} \mathrm{C}$ (left) and $700{ }^{\circ} \mathrm{C}$ (right). Bottom: corresponding graphs of the $\mathrm{B}$ concentration, with dark grey regions representing compact bulk boron, and light grey regions on the $\mathrm{B}$ layer surface representing less compact surface regions. At the B-to-Si interface the minus and plus signs represent respectively the fixed negative charge and the associated hole accumulation. Reprinted with permission from [18], Liu, X., et al., 2017. Nanometer-thin pure boron layers as mask for silicon micromachining. J. Micromechanical Systems, 26(6), pp.1428-1434. Copyright@IEEE. 
The sheet resistance, $R_{\text {sh }}$, along the B-to-Si interface is another parameter that gives important information on the electrical quality of the interface [42]. For lightly-doped $n$-Si substrates, the $R_{\text {sh }}$ of $700{ }^{\circ} \mathrm{C}$ PureB will be determined by B-doping of the bulk $\mathrm{Si}$ and it was found to be about $10 \mathrm{k} \Omega / \mathrm{sq}$. At $400{ }^{\circ} \mathrm{C}$ only the interfacial hole layer plays a role and $R_{\mathrm{sh}}$ increased to about $35 \mathrm{k} \Omega / \mathrm{sq}$. The behavior of the $R_{\mathrm{sh}}$ as a function of reverse voltage and substrate doping follows what would be expected of a hole inversion layer formed at the interface by an equally large concentration of fixed negative charge [41]. From extensive simulations based on this model it was concluded that without any metallization on the PureB an atomically-thin B coverage would be enough to achieve the attractively low electron current. This was confirmed by several experimental results including an experiment where lowelectron-current PureB diodes were successfully fabricated at $250{ }^{\circ} \mathrm{C}$ with a B-thickness of about $1 \mathrm{~nm}$ [7]. However, these diodes were not directly contacted by a metal layer. Simulations predict that an interfacial hole layer of concentrations above about $5 \times 10^{13} \mathrm{~cm}^{-2}$ can be responsible for the low saturation current reliably reproduced in the PureB diodes fabricated with B-deposition temperatures from $400{ }^{\circ} \mathrm{C}$ to $700{ }^{\circ} \mathrm{C}$. When a metal layer is added, the electron current may increase for $700{ }^{\circ} \mathrm{C}$ layers thinner than $1 \mathrm{~nm}$ because the metal work function starts to influence the electric field at the B-to-Si interface [7]. This has meant that with deposition temperatures below $400{ }^{\circ} \mathrm{C}$, where the B-layer is more loosely bonded, layers thicker than $4 \mathrm{~nm}$ are needed to avoid detrimental effects of the metallization.

The surface density of Si atoms on a (100) substrate is $6.78 \times 10^{14} \mathrm{~cm}^{-2}$. For an interfacial hole layer concentration around that value, simulations indicate that the metallization problem is much less critical. For advanced nanoscale diodes such as those studied for application in advanced (tunnelling) FETs [42], the high resistivity of the amorphous bulk B may become an issue. It has been measured to be from $500 \Omega-\mathrm{cm}$ to $10^{4} \Omega-\mathrm{cm}$, depending on the deposition conditions [30], and even a tunnelling-layer thickness of $2 \mathrm{~nm}$ to $3 \mathrm{~nm}$ represents a series resistance that could be limiting for device speed. Therefore, it may become crucial to realize an almost complete monolayer of fixed negative charge at low temperatures with less than 1-nm-thick PureB layers to comply with the series resistance specifications. Research in this direction is on-going.

For mm-large photodiode circuits, the $R_{\mathrm{sh}}$ can also become a limiting factor for the speed. For example, for the electron detector illustrated in Figure 1, the series resistance specification could not be reached without taking extra precautions. Therefore, to lower the resistance to the central photodiode surfaces, an Al-metal grid was patterned directly on the PureB surface. For this, resist patterning and $\mathrm{Al}$ removal by wet-etching in diluted $\mathrm{HF}$ selectively to the PureB was applied. Drive-in of the B at temperatures above $700{ }^{\circ} \mathrm{C}$ can also be used to reduce the series resistance but at the cost of added junction depth and possible damage of the PureB coverage [9].

PureB deposition on Si by CVD has up until now been limited to temperatures above $250{ }^{\circ} \mathrm{C}$, but with $\mathrm{MBE}$, PureB layers can be deposited on any surface at temperatures as low as $50{ }^{\circ} \mathrm{C}$ [14]. Unlike CVD layers, the MBE layers are not conformal and there is no selectivity to prevent deposition on the $\mathrm{SiO}_{2}$ surrounding the windows to the $\mathrm{Si}$. Nevertheless, for many other characteristics of PureB diodes, made by either CVD or MBE, the Si substrate temperature appears to be the decisive parameter. When comparing $300{ }^{\circ} \mathrm{C}-700{ }^{\circ} \mathrm{C}$ depositions, the electrical characteristics were similar and as was the compactness of the layers. Both features degraded as the substrate temperature was reduced but still, even at $50{ }^{\circ} \mathrm{C}$, the B formed diodes with $p^{+} n$-like $I-V$ characteristics.

The PureB diode research has stimulated interest in finding materials that could create similarly shallow $n^{+}$-type regions without actually doping the substrate. Obvious candidates are the n-dopant impurities As, $\mathrm{P}$ and $\mathrm{Sb}$, but as of yet no satisfactory results have been reported. Deposition of As and P on Si by CVD is readily achieved at low temperatures but self-limited monolayers are formed that are unstable in air. Suitable capping layers would need to be found before the electrical behavior of the resulting interface could be investigated properly. In the case of $\mathrm{Sb}$, preliminary experiments with deposition by $\mathrm{MBE}$ were performed but did not lead to any useful electrical results [14]. A point of concern is that these $n$-dopant materials do not display the same strength and stability as pure B so choosing a material to cap the interface layer before exposure to air or metallization layers will very likely have to be sought.

\section{BORON DEPOSITED ON NON-Si SEMICONDUCTORS}

Most of the PureB research has been focused on B depositions on $\mathrm{Si}$, but interesting results have also been achieved for layers used to fabricate $\mathrm{Ge}$ and $\mathrm{GaN}$ diodes. In both cases, the PureB was able to form a barrier to eliminate chemical reactions between an Al-metallization and the semiconductor. Even for metallization on $\mathrm{Si}$, metals like $\mathrm{Al}, \mathrm{Au}$ and $\mathrm{Cu}[43]$ react with the $\mathrm{Si}$ at the standard alloying temperature of $400{ }^{\circ} \mathrm{C}$, resulting in spiking or metal-Si interdiffusion that is detrimental to shallow junction formation [44]. At present the most commonly used barrier layer between $\mathrm{Al}$ and $\mathrm{Si}$ is $\mathrm{TiN}$, either sputtered for tens of nm thick layers or deposited by atomic layer deposition for thinner conformal layers [45]. TiN is also an important barrier layer in the processing of the most popular contacting layers to $\mathrm{Ge}$ and $\mathrm{GaN}$, both of which are much more instable than $\mathrm{Si}$ in the presence of metals and much less tolerant to temperature steps. 

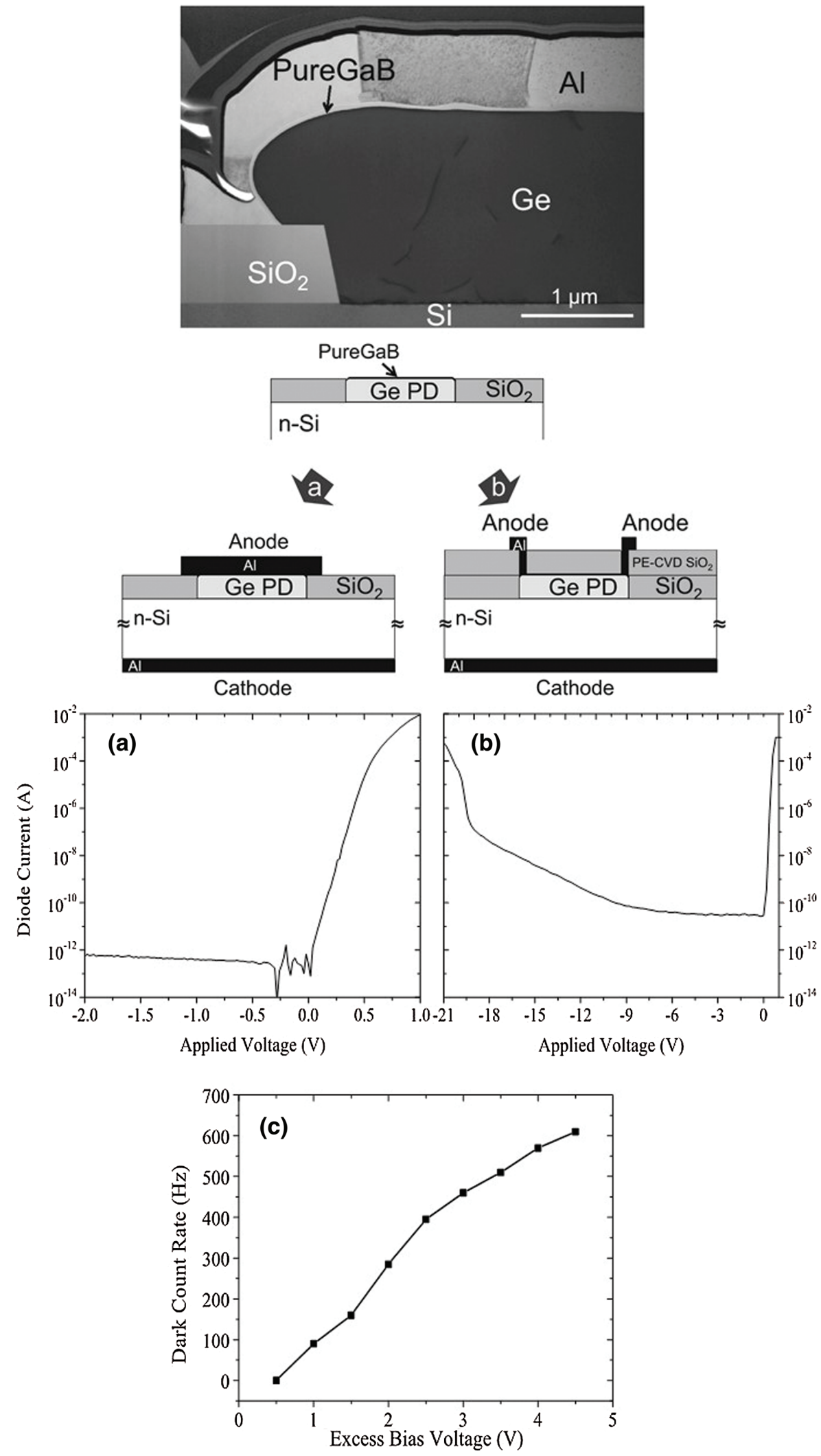

Figure 5. Top: TEM image of a Ge-on-Si island fabricated with a PureGaB barrier layer to Al metallization. Reprinted with permission from [47], Sammak, A., et al., 2015. Restricted-access Al-mediated material transport in al contacting of PureGaB Ge-on-Si $p^{+} n$ diodes. Journal of Electronic Materials, 44(12), pp.4676-4883. Copyright@Springer. Middle: Schematic process flow for the fabrication of PureGaB Ge-on-Si diodes with two different metallization methods. Bottom: (a) and (b) $I-V$ characteristics of a $5 \times 5 \mu \mathrm{m}^{2}$ PureGaB Ge-on-Si diode, and (c) the dark count rate for Geiger mode operation of this diode. 
$\mathrm{Ge}$ is a group IV semiconductor similar to $\mathrm{Si}$ and $\mathrm{B}$ is also a common dopant material in Ge. Presumably due to the low boron solid solubility in Ge, PureB deposition did not readily provide an interfacial $p^{+}$-like region as observed on $\mathrm{Si}$. In contrast, Ga has a high solid solubility in Ge of above $10^{20} \mathrm{~cm}^{-3}$ for temperatures above $100{ }^{\circ} \mathrm{C}$ [46]. A $p^{+} n$-like diode was achieved on the Ge by first depositing a layer of pure $\mathrm{Ga}$ and covering with a layer of PureB to both prevent oxidation of the $\mathrm{Ga}$ and to create a barrier to the Al metallization [47]. With this PureGaB process module, near-ideal Ge-on-Si diodes were fabricated with very low saturation currents, and abrupt reverse breakdown characteristics. When tested as near-infrared photodiodes these characteristics were ideal enough to allow operation in Geiger mode at room temperature with very low dark count rate [16], an example of which is shown in Figure 5.

In GaN HEMTs, Ni-Au is the preferred metal stack for fabricating Schottky-diode gates, and stacks of TiN/Al/Ti are often used as contacts. Nevertheless, both metallization schemes have reliability issues, and Au is not CMOS compatible, so new metallization methods are still very actively being sought after [48].
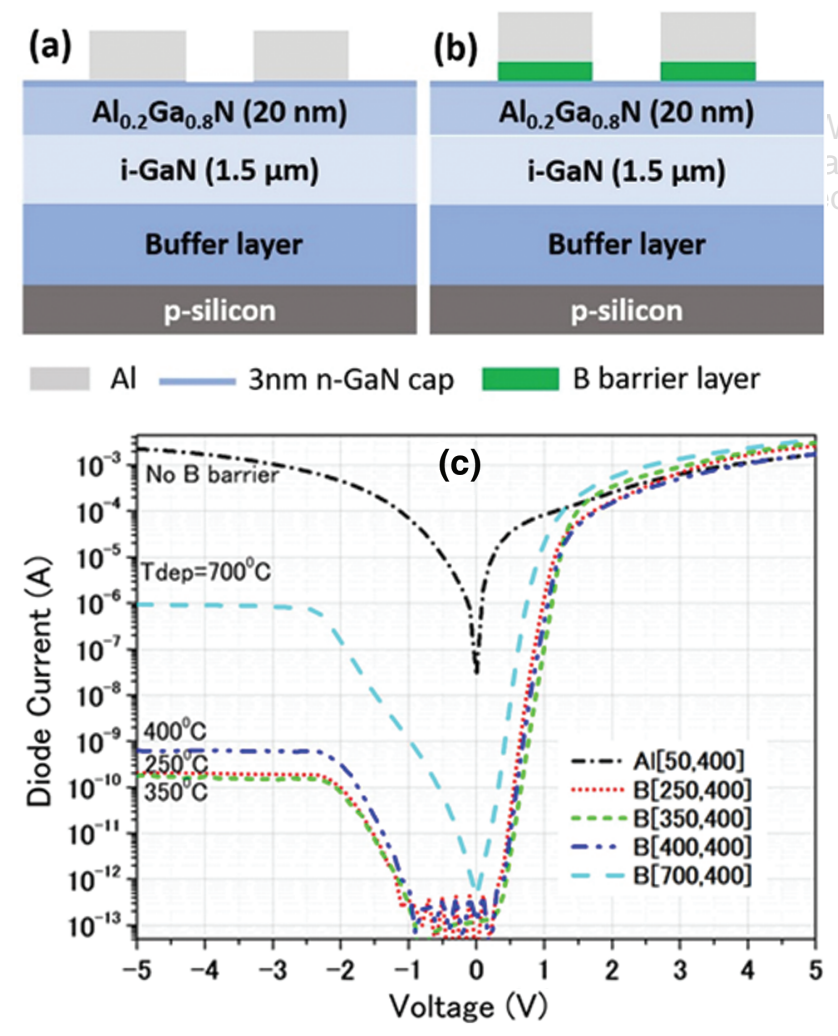

Figure 6. Schematic cross sections of the fabricated GaN diodes without (a) and with (b) a PureB barrier layer to the Al metallization. (c) $I-V$ characteristics of the fabricated diodes after annealing at $400{ }^{\circ} \mathrm{C}$. Reprinted with permission from [17], Thammaiah, S.D., et al., 2019. Nanometer-Thin Pure B Layers Grown by MBE as Metal Diffusion Barrier on GaN Diodes. 2019 China Semiconductor Technology International Conference, Shanghai, China, pp.1-3. Copyright@IEEE.
With low-temperature MBE PureB deposition as barrier layer to the $\mathrm{GaN}$, it was possible to fabricate Al-contacted diodes to $n$-type GaN with low saturation currents and stable behavior upon annealing at $400{ }^{\circ} \mathrm{C}$ [17]. As shown in Figure 6, the best results were achieved for $350{ }^{\circ} \mathrm{C}-400{ }^{\circ} \mathrm{C}$ $\mathrm{B}$ deposition. For an even lower deposition temperature of $250{ }^{\circ} \mathrm{C}$ a post-deposition anneal at $400{ }^{\circ} \mathrm{C}$ was beneficial in reducing the current level to that of the $350{ }^{\circ} \mathrm{C}-$ $400{ }^{\circ} \mathrm{C}$ diodes. This suggests that there may be a favorable B-to-GaN chemistry at $350{ }^{\circ} \mathrm{C}-400{ }^{\circ} \mathrm{C}$ in addition to the $\mathrm{nm}$-thin B-layers being a barrier to $\mathrm{Al}$ metallization.

\section{CONCLUSION}

PureB is a uniquely versatile material in having a deposition window from $50{ }^{\circ} \mathrm{C}$ to $700{ }^{\circ} \mathrm{C}$ for ultra-shallow $p^{+} n$-like diode formation. For these diodes, the PureB can also form a very stable and robust nm-thin capping layer that can function as a barrier to metals and several commonly used wet-etching steps. These are properties that have also made it a potentially potent material for MEMS processing and applications such as protection membranes. Despite great interest in finding a similarly reliable processes for the fabrication of interfacially-formed $n^{+} p$-like diodes, deposition of $n$-type dopant materials such as As, $\mathrm{P}$, and $\mathrm{Sb}$, have not yet lead to any viable solutions, among others because robust surface layers are not automatically formed.

Acknowledgments: The current work was in part financially supported by the Memphis II Project No. 13535 of The Netherlands Organisation for Scientific Research (NWO) Domain Applied and Engineering Sciences (TTW), and the Danish Innovation foundation for funding through the project "Semiconductor Materials for Power Electronics" (SEMPEL).

\section{References and Notes}

1. Zou, L., Wang, L., Wu, Y., Ma, C., Yu, S. and Liu, X, 2018. Trends analysis of graphene research and development. Journal of Data and Information Science, 3(1), pp.82-100.

2. Tao, L., Cinquanta, E., Chiappe, D., Grazianetti, C., Fanciulli, M., Dubey, M., Molle, A. and Akinwande, D., 2015. Silicene field-effect transistors operating at room temperature. Nature Nanotechnology, 10, pp.227-230.

3. Beilstein, J., 2018. Silicene, germanene and other group IV 2D materials. J. Nanotechnology, 9, pp.2665-2667.

4. Conley, H.J., Wang, B., Ziegler, J.I., Haglund, Jr., R.F., Pantelides, S.T. and Bolotin, K.I., 2013. Bandgap engineering of strained monolayer and bilayer $\mathrm{MoS}_{2}$. Nano Letters, 13(8), pp.3626-3630.

5. Liu, H., Neal, A.T., Zhu, Z., Tomanek, D. and Ye, P.D., 2014. Phosphorene: A new 2D material with high carrier mobility. ACS Nano, 8, pp.4033-4041.

6. Wang, Z.-Q., Lü, T.-Y., Wang, H.-Q., Feng, Y.P. and Zheng, J.-C., 2019. Review of borophene and its potential applications. Front Phys., 14(2), pp.1-45.

7. Knežević, T., Liu, X., Hardeveld, E., Suligoj, T. and Nanver, L.K., 2019. Limits on thinning of boron layers with/without metal contacting in PureB Si (photo) diodes. IEEE Electron Device Letters, 40(6), pp.858-861. 
8. Shi, L., Nihtianov, S., Haspeslagh, L., Scholze, F., Gottwald, A. and Nanver, L.K., 2012. Surface-Charge-Collection-Enhanced HighSensitivity High-Stability Silicon Photodiodes for DUV and VUV Spectral Ranges. IEEE Transactions on Electron Devices, 59(11), pp.2888-2894.

9. Nanver, L.K., Qi, L., Mohammadi, V., Mok, K.R.M., De Boer, W.B., Golshani, N., Sammak, A., Scholtes, T.L.M., Gottwald, A., Kroth, U. and Scholze, F., 2014. Robust UV/VUV/EUV PureB photodiode detector technology with high CMOS compatibility. IEEE J. Selected Topics in Ouantum Electronics, 20(6), Art no. 3801711.

10. Šakić, A., Nanver, L.K., van Veen, G., Kooijman, K., Vogelsang, P., Scholtes, T.L.M., de Boer, W., Wien, W.H.A., Milosavljevic, S., Heerkens, C.Th.H., Knežević, T. and Spee, I., 2010. Versatile Silicon Photodiode Detector Technology for Scanning Electron Microscopy with High-Efficiency sub-5 keV Electron Detection. Proc. 2010 IEEE Intl. Electron Devices Meeting, Washington, DC, USA, pp.31.4.1-31.4.4.

11. Kessler, M.A., Ohrdes, T., Wolpensinger, B., Bock, R. and Harder, N.-P., 2010. Charge carrier lifetime degradation in $\mathrm{Cz}$ silicon through the formation of a boron-rich layer during $\mathrm{BBr}_{3}$ diffusion processes. Semiconductor Science and Technology, 25(5), pp.55001-55009.

12. Phang, S.P., Liang, W., Wolpensinger, B., Kessler, M.A. and Macdonald, D., 2013. Tradeoffs between impurity gettering, bulk degradation and surface passivation of boron-rich layers on silicon solar cells. IEEE J. Photovoltaics, 3(1), pp.261-266.

13. Mok, K.R.C., van de Loo, B.W.H., Vlooswijk, A.H.G., Kessels, W.M.M. and Nanver, L.K., 2015. Boron-doped silicon surfaces from $\mathrm{B}_{2} \mathrm{H}_{6}$ passivated by $\mathrm{ALD} \mathrm{Al}_{2} \mathrm{O}_{3}$ for solar cells. IEEE J. Photovoltaics, 5(5), pp.1310-1318.

14. Knežević, T., Elsayed A., Dick, J.F., Liu, X., Schulze, J., Suligoj, T. and Nanver, L.K., 2019. Back-end-of-line CMOS-compatible diode fabrication with pure boron deposition down to $50{ }^{\circ} \mathrm{C}$. Proc. 49th European Solid-State Device Research Conf., Krakow, Polen, pp.1-3.

15. Calvert, J.B., 2009. Boron. University of $D$ Denver (http://mysite.du.edu/jcalvert/phys/boron.htm).

16. Sammak, A., Aminian, M., Qi, L., De Boer, W.B., Charbon, E. and Nanver, L.K., 2011. A CMOS Compatible Ge-on-Si APD Operating in Proportional and Geiger Modes at Infrared Wavelengths. Proc. IEEE Intl. Electron Devices Meeting, Washington, DC, USA, pp.8.5.1-8.5.4.

17. Thammaiah, S.D., Lundsgaard Hansen, J. and Nanver, L.K., 2019 Nanometer-Thin Pure B Layers Grown by MBE as Metal Diffusion Barrier on GaN Diodes. 2019 China Semiconductor Technology International Conference (CSTIC), 18-19 March; Shanghai, China, pp.1-3.

18. Liu, X., Nanver, L.K. and Scholtes, T.L.M., 2017. Nanometer-thin pure boron layers as mask for silicon micromachining. J. Micromechanical Systems, 26(6), pp.1428-1434.

19. Widom, M. and Mihalkovič, M., 2008. Symmetry-broken crystal structure of elemental boron at low temperature. Physical Review B, 77(6), Article No. 064113.

20. Johansson, S., Schweitz, J.-Å., Westberg, H. and Boman, M., 1992. Microfabrication of three-dimensional boron structures by laser chemical processing. Journal of Applied Physics, 72(12), pp.59565963.

21. Sarubbi, F., Nanver, L.K. and Scholtes, T.L.M., 2010. High effective gummel number of CVD boron layers in ultrashallow $p^{+} n$ diode configurations. IEEE Transactions on Electron Devices, 57(6), pp.1269-1278.

22. Mohammadi, V., de Boer, W.B. and Nanver, L.K., 2012. Temperature dependence of chemical-vapor deposition of pure boron layers from diborane. Applied Physics Lett., 101(11), p.111906.

23. Shi, L., Nanver, L.K. and Nihtianov, S.N., 2011. Stability characterization of high-sensitivity silicon-based EUV photodiodes in a detrimental industrial environment. Proc. 37th Annual Conference of the IEEE Industrial Electronics Society, Melbourne, VIC, Australia, pp.2651-2656.

24. Inkson, B.J., 2016. in Material Characterisation Using Nondestructive Evaluation Methods, Woodhead Publishing, pp.17-43.

25. Qi, L., Sluyterman, S., Kooijman, K., Mok, K.R.C. and Nanver, L.K., 2015. PureB single-photon avalanche diodes for lowenergy electron detection down to $200 \mathrm{eV}$. Optics Letters, 40(3), pp.300-303

26. Elwenspoek, M. and Jansen, H.V., 1998. Silicon Micromachining, Cambridge Univ. Press.

27. Liu, X., Italiano, J., Scott, R. and Nanver, L.K., 2019. Micromachined nanometer-thin boron membranes on silicon. Materials Research Express, 6(11), p.116438.

28. Stoll, J.D. and Kolmakov, A., 2012. Electron transparent graphene windows for environmental scanning electron microscopy in liquids and dense gases. Nanotechnology, 23(505704), pp.1-8.

29. Han, Y., Nguyen, K., Ogawa, Y., Shi, H., Park, J. and Muller, D.A., 2015. Electron microscopy in air: Transparent atomic membranes and imaging modes. Microscopy and Microanalysis, 21(Suppl 3), pp.1111-1112.

30. Mohammadi, V., Ramesh, S. and Nanver, L.K., 2014. Thickness Evaluation of Deposited PureB Layers in Micro-/Millimeter-Sized Windows to Si. Proc. 27th Intl. Conf. Microelectronic. Test Structures, pp.194-199.

31. Shi, L., Nihtianov, S., Xia, S., Nanver, L.K., Gottwald, A. and Scholze, F., 2012. Electrical and Optical Performance Investigation of Si-Based Ultrashallow-Junction $p^{+}-n$ VUV/EUV Photodiodes IEEE Trans. Instrum. Meas., 61(5), pp.1268-1277.

32. van Zwol, P.J., Nasalevich, M., Kurganova, E., Voorthuijzen, P. Vles, D., Péter, M., Symens, W., van der Zande, W., Notenboom, A., Klootwijk, J., van de Kruijs, R.W. and Giesbers, A.J.M., 2017. Pellicle films supporting the ramp to HVM with EUV. Proc. of SPIE, 10451, pp.1-9.

33. Henke, B.L., Gullikson, E.M. and Davis, J.C., 1993. X-ray interactions: Photoabsorption, scattering, transmission, and reflection at $E=50-30,000$ eV, Z =1-92. Atomic Data Nucl. Data Tables, 54 , pp.181-342.

34. Mannix, A.J., Zhou, X.-F., Kiraly, B., Wood, J.D., Alducin, D. Myers, B.D., Liu, X., Fisher, B.L., Santiago, U., Guest, J.R., Yacaman, M.J., Ponce, A., Oganov, A.R., Hersam, M.C. and Guisinger, N.P., 2015. Synthesis of borophenes: Anisotropic, twodimensional boron polymorphs. Science, 350-6267, pp.1513-1516.

35. Wu, R., Drozdov, I.K., Eltinge, S., Zahl, P., Ismail-Beigi, S., Božović, I. and Gozar, A., 2019. Large-area single-crystal sheets of borophene on $\mathrm{Cu}(111)$ surfaces. Nature Nanotechnology, 14, pp.44-49.

36. Piazza, Z.A., Hu, H.-S., Li, W.-L., Zhao, Y.-F., Li, J. and Wang, L.-S., 2014. Planar hexagonal B36 as a potential basis for extended single-atom layer boron sheets. Nature Communications, 5(3113), pp.1-6.

37. Zhang, Z., Penev, E.S. and Yakobson, B.I., 2017. Two-dimensional boron: structures, properties and applications. Chem. Soc. Rev., 46 , pp.6746-673.

38. Nanver, L.K., Lyon, K., Liu, X., Italiano, J. and Huffman, J., 2018. Material reliability of low-temperature boron deposition for pureB silicon photodiode fabrication. MRS Advances, 3(57-58), pp.33973402 .

39. Sammak, A., Qi, L., de Boer, W.B. and Nanver, L.K., 2010. Chemical Vapor Deposition of Ga Dopants for Fabricating Ultrashallow pn Junctions at $400{ }^{\circ} \mathrm{C}$. Proceedings of 10th IEEE Intl. Conf. Solid-State and Integrated Circuit Technology, pp.969-971.

40. Nanver, L.K., 2018. An Experimental View on PureB Silicon Photodiode Device Physics. Proc. 41st International Convention on Information and Communication Technology, Electronics and Microelectronics, pp.0001-0006. 
41. Qi, L. and Nanver, L.K., 2015. Conductance Along the Interface Formed by $400{ }^{\circ} \mathrm{C}$ Pure Boron Deposition on Silicon. IEEE Electron Device Lett., 36(2), pp.102-104.

42. Llorente, C.D., Martinie, S., Cristoloveanu, S., Colinge, J.-P., Le Royer, C., Wan, J., Ghibaudo, G. and Vinet, M., 2018. Innovative tunnel FET architectures. in 2018 Joint Intl. EUROSOI-ULIS Workshop, pp.1-4.

43. Zenou, V.Y., Kiv, A., Fuks, D., Ezerski, V. and Moiseenko, N., 2006. The microscopic mechanism of silicon precipitation in $\mathrm{Al} / \mathrm{Si}$ system. Materiasl Science and Engineering A, 435-436, pp.556-563.

44. Lee, H.-J., Sinclair, R., Li, P. and Roberts, B., 1999. A study of the failure mechanism of a titanium nitride diffusion barrier. Journal of Applied Physics, 86(6), pp.3096-3103.
45. Kim, D.-J., Jung, Y.-B., Lee, M.-B., Lee, Y.-H. and Lee, J.-H., 2000. Applicability of ALE TiN films as $\mathrm{Cu} / \mathrm{Si}$ diffusion barriers. Thin Solid Films, 372, pp.276-283.

46. Trumbore, F.A., 1960. Solid solubilities of impurity elements in germanium and silicon. Bell System Technical Journal, 39(1), pp.205233.

47. Sammak, A., Qi, L. and Nanver, L.K., 2015. Restricted-access Almediated material transport in $\mathrm{Al}$ contacting of pureGaB Ge-on-Si $p+n$ diodes. Journal of Electronic Materials, 44(12), pp.46764883.

48. Hajlasz, M., Donkers, J.J.T.M., Sque, S.J., Heil, S.B.S., Gravesteijn, D.J., Rietveld, F.J.R. and Schmitz, J., 2014. Sheet resistance under ohmic contacts to AlGaN/GaN heterostructures. Applied Physics Letters, 104(242109), pp.1-5.

Received: 16 January 2020. Accepted: 25 May 2020. 